PROCEEDINGS OF THE

AMERICAN MATHEMATICAL SOCIETY

Volume 125, Number 10, October 1997, Pages 2819-2824

S 0002-9939(97)04216-0

\title{
ON THE NUMBER OF COMPONENTS OF THE MODULI SCHEMES OF STABLE TORSION-FREE SHEAVES ON INTEGRAL CURVES
}

\author{
E. BALLICO
}

(Communicated by Eric Friedlander)

\begin{abstract}
Here we give an upper bound for the number of irreducible components of the moduli scheme of stable rank $r$ torsion-free sheaves of fixed degree on the integral curve $X$. This bound depends only on $r, \operatorname{Sing}(X), p_{a}(X)$ and the corresponding number for the rank 1 case.
\end{abstract}

Let $X$ be an integral complete curve of arithmetic genus $p_{a}$ and geometric genus $g$. For all integers $r, d$ with $r \geq 1$ let $M(X, r, d)$ (or just $M(r, d)$ ) be the moduli scheme of stable rank $r$ torsion-free sheaves on $X$ with degree $d$. For general background on stable vector bundles and sheaves on curves and their moduli schemes, see $[\mathrm{N}]$ or $[\mathrm{S}]$. The number of irreducible components of $M(r, d)$ does not depend on $d$, but only on $X$ and $r$ (see Theorem 1.2). Let $n(r, X)$ (or just $n(r)$ ) be the number of irreducible components of $M(r, d)$. Here we want to give upper bounds on $n(r)$ depending only on the singularities of $X$, the integer $r$ and $n(1)$. This is given by Theorem 0.1 (see the discussion in the last part of Remark 1.4). To state Theorem 0.1 (our main result) we need to introduce a few concepts (e.g. measures for the singularities of $X$ ) and fix some notation.

Let $\pi: C \rightarrow X$ be the normalization (hence $g=p_{a}(C)$ ). Set $\delta:=p_{a}-g$. Set $\boldsymbol{O}:=\boldsymbol{O}_{X}$. For any $x \in \operatorname{Sing}(X)$, set $\boldsymbol{O}_{x}:=\boldsymbol{O}_{X, x}$ and let $\mathbf{m}_{x}$ be the maximal ideal of $\boldsymbol{O}_{x} ;$ set $\mathbf{k}_{x}:=\boldsymbol{O}_{x} / \mathbf{m}_{x}$. Set $f_{x}:=\operatorname{dim}_{\mathbf{K}}\left(\operatorname{Ext}^{1}\left(\mathbf{k}_{x}, \boldsymbol{O}_{x}\right)\right)$ and $\mathbf{f}:=\max \left\{f_{x}\right\}_{x \in \operatorname{Sing}(X)}$. For example, we have $f_{x}=1$ if and only if $\boldsymbol{O}_{x}$ is Gorenstein (see e.g. [Co], Lemma 2.1.4). Let $e_{x}$ be the multiplicity of $X$ at $x$; set $e:=\max \left(e_{x}\right)$ and $e^{\prime \prime}:=\sum_{x} e_{x}$. Let $F$ be a rank $r$ torsion-free sheaf on $X$; the set of all formal isomorphism classes of the germs $\left\{F_{x}\right\}_{x \in \operatorname{Sing}(X)}$ will be called the formal isomorphism class of $F$. The set $W$ of all possible isomorphism classes for rank $r$ torsion-free sheaves around $\operatorname{Sing}(X)$ has in a natural way a scheme structure; for any reduced subscheme $\Omega$ of $W$, let $M(r, d, \Omega)$ be the reduced subscheme of $M(r, d)_{\text {red }}$ parametrizing sheaves with formal isomorphism type in $\Omega$. Let $n(r, \Omega)$ (or $n(r, d, \Omega)$ ) be the number of irreducible components of $M(r, d, \Omega)$. By Theorem 1.2 this integer does not depend on $d$. Our main interest is when $\Omega$ is an irreducible component of $W_{\text {red }}$.

Here is our main result.

Received by the editors November 28, 1994.

1991 Mathematics Subject Classification. Primary 14H60, 14D20, 14 B99.

This research was partially supported by MURST and GNSAGA of CNR (Italy). The author is a member of Europroj (and its group "Vector bundles on curves").

(C)1997 American Mathematical Society 
Theorem 0.1. Fix an irreducible component, $\Gamma$, of the scheme of formal isomorphism types of rank $r$ torsion-free sheaves around $\operatorname{Sing}(X)$. Let $n(r, \Gamma)(r \geq 1)$ be the number of irreducible components of the moduli scheme of rank $r$ torsion-free sheaves on $X$ with fixed degree and with formal isomorphism type in $\Gamma$. Then for every $r \geq 2$, we have:

$$
n(r, \Gamma) \leq(r-1)\left(e^{\prime \prime}(e-1)+(r+1)\left(2 p_{a}-2+e\right)\right) n(1)+r+r \delta f .
$$

The main steps in the proof of Theorem 0.1 are the proof of Theorem 1.2 and of the Construction 1.3. In our opinion the statement of Theorem 1.2 has independent interest.

\section{THE PROOFS}

Here we prove Theorem 0.1, Theorem 1.2 and a related Remark. We recall the following constructions. Fix an integer $r>1$, an integral projective curve $X$, $P \in X_{\text {reg }}, Q \in X_{\text {reg }}$ and a torsion-free sheaf $M$ on $X$ with $\operatorname{rank}(M)=r$. Let $\mathbf{k}_{P}$ be the skyscraper length 1 sheaf on $X$ supported by $P$. Fix any surjection $t: M \rightarrow \mathbf{k}_{P}$ and any surjection $j: M(Q) \rightarrow\left(\mathbf{k}_{Q}\right)^{\oplus(r-1)}$. Since $P$ and $Q$ are smooth points of $X$, they are Cartier divisors on $X$ and $M$ is locally free at $P$ and $Q$. Hence (see e.g. $[\mathrm{M}]), \operatorname{Ker}(t)$ and $\operatorname{Ker}(j)$ are locally free on $X_{\text {reg }}$ and have the same singularity type of $M$ at each point of $\operatorname{Sing}(X)$. We will say that the sheaf $\operatorname{Ker}(t)$ is obtained from $M$ by an elementary transformation supported by $P$ and that $\operatorname{Ker}(j)$ is obtained from $M$ by an elementary antitransformation supported by $Q$. Note that $\operatorname{rank}(\operatorname{Ker}(t))=\operatorname{rank}(\operatorname{Ker}(j))=r, \operatorname{deg}(\operatorname{Ker}(t))=\operatorname{deg}(M)-1$ and $\operatorname{deg}(\operatorname{Ker}(j))=\operatorname{deg}(M)+1$. Note that $M$ is obtained from $\operatorname{Ker}(t)$ by an elementary antitransformation supported by $P$ and from $\operatorname{Ker}(j)$ by an elementary transformation supported by $Q$. If $M^{\prime \prime}$ (resp. $M^{\prime}$ ) is obtained from $M$ by an elementary transformation (resp. elementary antitransformation) supported by $P$, then $\mathcal{H o m}\left(M, \omega_{X}\right)$ is obtained from $\mathcal{H o m}\left(M^{\prime \prime}, \omega_{X}\right)\left(\right.$ resp. $\left.\mathcal{H o m}\left(M^{\prime}, \omega_{X}\right)\right)$ by an elementary antitransformation (resp. elementary transformation) supported by $P$.

Lemma 1.1. Fix $X, r, d$ and $y$ such that $r$ divides $d-y$ and a reduced family $\Omega$ of formal isomorphism types for $\operatorname{Sing}(X)$. Then the schemes $M(X, r, d)_{\text {red }}$ and $M(X, r, y)_{\mathrm{red}}\left(r e s p . M(r, d, \Omega)_{\mathrm{red}}\right.$ and $\left.M(r, y, \Omega)_{\mathrm{red}}\right)$ have the same number of irreducible components.

Proof. Choose $P \in X_{\text {reg }}$. For every $E \in M(X, r, y)$, the sheaf

$$
E \otimes \boldsymbol{O}_{X}(((d-y) / r) P) \in M(X, r, d)
$$

and has the same isomorphism type as $E$. The induced bijection can be done even if $E$ moves in a flat family. Hence if $r$ divides $d-y$, the schemes $M(X, r, d)$ and $M(X, r, y)$ (resp. $M(r, d, \Omega)$ and $M(r, y, \Omega)$ ) are isomorphic, being coarse moduli spaces of equivalent functors.

Theorem 1.2. For every $X, r, d$ and $y$ the schemes $M(X, r, d)_{\mathrm{red}}$ and $M(X, r, y)_{\mathrm{red}}$ have the same number of irreducible components. Furthermore, for any reduced family $\Omega$ of formal isomorphism types for $\operatorname{Sing}(X)$ the schemes $M(r, d, \Omega)_{\mathrm{red}}$ and $M(r, y, \Omega)_{\text {red }}$ have the same number of irreducible components.

Proof. By Lemma 1.1 we reduce easily to the case $r>1, d=y+1$. Choose $P \in X_{\text {reg. }}$. Consider the contravariant functor $\Lambda$ which to a scheme $S$ associates a rank $r$ sheaf $\Phi$ on $S \times X$ flat over $S$ plus an isomorphism of $\Phi \mid S \times\{P\}$ with 
the trivial rank $r$ sheaf. Add to this functor the stability condition and that the relative degree over $S$ is $d$ (resp. $y$ ). Fix $F \in M(X, r, d)$ and consider an elementary transformation $F^{\prime \prime}$ of $F$ supported by $P . F^{\prime \prime}$ has rank $r$ and degree $y$. We want to check that in this way we get a bijection of the set of irreducible components of the schemes $M(X, r, d)_{\text {red }}$ and $M(X, r, y)_{\text {red. }}$. Fix an irreducible component $T$ of $M(X, r, d)_{\text {red }}$ and a general sheaf $F \in T$. We want to check that, in general, the corresponding $F^{\prime \prime}$ is stable. Since $F$ is stable and $\operatorname{deg}\left(F^{\prime \prime}\right)=\operatorname{deg}(F)-1$, every proper subsheaf $B$ of $F^{\prime \prime}$ with $r \chi_{B} \geq \operatorname{rank}(B) \chi_{F^{\prime \prime}}$ (if any) is saturated in $F$ and in particular it is saturated in $F^{\prime \prime}$. Assume that this is not the case and let $u$ be the minimal integer with $0<u<r$ and such that there is a rank $u$ saturated subsheaf $A$ of $F^{\prime \prime}$ with $r \chi_{A} \geq \operatorname{rank}(A) \chi_{F^{\prime \prime}}$. Consider the set $\Sigma$ of all rank $u$ subsheaves of $F$ with $\chi_{A}$ as Hilbert polynomial. By the minimality of $u$ every $A \in \Sigma$ is stable. Take $A^{\prime}, A^{\prime \prime} \in \Sigma$ and look at the image $A^{\prime}+A^{\prime \prime}$ of $A^{\prime} \oplus A^{\prime \prime}$ for the natural map $m: A^{\prime} \oplus A^{\prime \prime} \rightarrow F^{\prime \prime}$. If $A^{\prime} \neq A^{\prime \prime}$ as subsheaves of $F^{\prime \prime}$, we have $\operatorname{rank}(\operatorname{Ker}(m))<u$. Since $\chi_{\operatorname{Ker}(m)}+\chi_{\operatorname{Im}(u)}=\chi_{A^{\prime} \oplus A^{\prime \prime}}=2 \chi_{A^{\prime}}$, if $A^{\prime} \neq A^{\prime \prime}$ as subsheaves of $F^{\prime \prime}$ we have $A^{\prime}+A^{\prime \prime} \cong A^{\prime} \oplus A^{\prime \prime}$ by the minimality of the integer $u$. Note that if $A^{\prime} \neq A^{\prime \prime}$, then $A^{\prime} \oplus A^{\prime \prime}$ is saturated in $F^{\prime \prime}$ and it is saturated in $F$ if $A^{\prime} \oplus A^{\prime \prime} \neq F^{\prime \prime}$. Fix $B \in \Sigma$. If $A^{\prime}+A^{\prime \prime}+B \neq A^{\prime}+A^{\prime \prime}$ in the same way we see that $A^{\prime}+A^{\prime \prime}+B \cong A^{\prime} \oplus A^{\prime \prime} \oplus B$, that $A^{\prime}+A^{\prime \prime}+B$ is saturated in $F^{\prime \prime}$ and that it is saturated in $F$ unless $A^{\prime}+A^{\prime \prime}+B=F^{\prime \prime}$. If $A^{\prime}+A^{\prime \prime}+B=A^{\prime}+A^{\prime \prime} \cong A^{\prime} \oplus A^{\prime \prime}$, projecting onto the factor $A^{\prime}$ we see that either $B \cong A^{\prime}$ or $B \cong A^{\prime \prime}$. Then we continue. We obtain a maximal integer $k \geq 1$ such that there are $A_{j} \in \Sigma, 1 \leq j \leq k$, with $G:=A_{1}+\cdots+A_{k} \cong A_{1} \oplus \cdots \oplus A_{k}, G$ saturated in $F^{\prime \prime}$ and for every $B \in \Sigma$ we have $B \subseteq G$ and $B \cong A_{b}$ for some integer $b \leq k$. Furthermore, if $A_{1}, \ldots, A_{t}$ are not isomorphic as abstract sheaves, either $B=A_{i}$ (as subsheaves of $F^{\prime \prime}$ ) for some $i \leq t$ or $A_{1}+\cdots+A_{t}+B \neq A_{1}+\cdots+A_{t}$. Hence $\Sigma$ has only finitely many, say $s$, isomorphic classes of sheaves. Furthermore, up to reordering $\{1, \ldots, k\}$, we have $G=B_{1}+\cdots+B_{s}$ with $B_{j} \cong r_{j} A_{j}, r_{1}+\cdots+r_{s}=k u$, and every $A \in \Sigma$ is contained in some $B_{j}$. Consider an elementary antitransformation

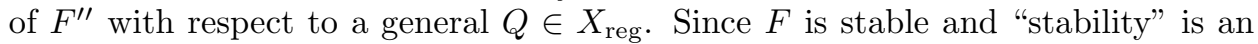
open condition, we see that a general such $H$ is stable and (since $X$ and $G L(r)$ are integral) $H \in T$. Note that $F^{\prime \prime} \subset H$. Hence we see that the only rank $u$ sheaves with the same Hilbert polynomial of $A_{1}$ and contained in a general such $H$ are the ones contained in $F^{\prime \prime}$. Take $H$ instead of $F$ and another general point $P^{\prime}$ to make the elementary transformation. Let $H^{\prime \prime} \subset H$ with length $\left(H / H^{\prime \prime}\right)=1$ be the kernel of this elementary transformation. A subsheaf $U$ of $H$ is a subsheaf of $H^{\prime \prime}$ if and only if the fiber $U \mid\left\{P^{\prime}\right\}$ of $U$ over $P^{\prime}$ is contained in the hyperplane of the fiber $H \mid\left\{P^{\prime}\right\}$ which is the kernel over $P^{\prime}$ of the elementary transformation. Thus we see that for general $F \in T$ we have $s=1, r=k u$ (hence $k>1$ ) and $F^{\prime \prime} \cong k A_{1}$. However, since $k>1$, twisting one of the factors of $k A_{1}$ by a general element of $\operatorname{Pic}(X)$ we obtain easily nearby sheaves for which the condition " $F$ " $\cong k A_{1}$ for some stable rank $u A_{1}$ " is not satisfied, a contradiction.

Note that the set of elementary transformations of a fixed rank $r$ torsion-free sheaf supported by a fixed point of $X$ is parametrized by an open subset of $\mathbf{K}^{r}$ and in particular it is irreducible. The same assertion is true for the set of elementary antitransformations. Since $X$ is integral, $T \times X \times \mathbf{K}^{r}$ is integral.

We have checked the existence of an open subset $L$ of $T \times X \times \mathbf{K}^{r}, L \neq \varnothing$, and a morphism $\mathbf{u}: L \rightarrow M(X, r, y)$. Since $L$ is integral, $\mathbf{u}(L)$ is contained in an irre-

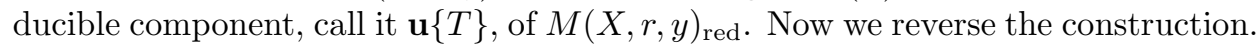


We start with an irreducible component $J$ of $M(X, r, y)_{\text {red }}$ and for every $G \in J$ we consider the torsion-free sheaves obtained from $G$ making an elementary antitransformation supported by some $P \in X_{\text {reg. }}$. We want to check that for fixed $P \in X_{\text {reg }}$, a general $G \in J$ and a general elementary antitransformation of $G$ supported by $P$, the corresponding sheaf $G^{\prime}$ is stable. Assume that this is not the case. Let $u$ be the minimal integer with $0<u<r$ and such that there is a rank $u$ subsheaf $A$ of $G^{\prime}$ with $r \chi_{A} \geq u \chi_{G^{\prime}}$. The minimality of $u$ implies that $A$ is stable. Since $G$ is a stable subsheaf of $G^{\prime}$ with $\operatorname{deg}(G)=\operatorname{deg}\left(G^{\prime}\right)-1$ we have $A \cap G \neq A$ and $A \cap G$ is saturated in $G$. Since $P \in X_{\text {reg }}$ this implies that $A$ is saturated in $G$. Now we may copy the proof given for the general elementary transformation of the general sheaf of the component $T$ of $M(X, r, d)_{\text {red }}$ and obtain a contradiction, i.e. the stability of $G^{\prime}$. In this way we found an open subset $L^{\prime}$ of $J \times X \times \mathbf{K}^{r}, L^{\prime} \neq \varnothing$, and a morphism $\mathbf{v}: L^{\prime} \rightarrow M(X, r, d)$. Since $L^{\prime}$ is integral, $\mathbf{v}(J)$ is contained in a unique irreducible component, $\mathbf{v}\{J\}$, of $M(X, r, d)_{\text {red }}$. Since the inverse of an elementary transformation is an elementary antitransformation we have $\mathbf{u}\{\mathbf{v}\{J\}\}=J$ and $\mathbf{v}\{\mathbf{u}\{T\}\}=T$ for all $J, T$. In this way we obtain a bijection between the irreducible components of $M(X, r, d)_{\text {red }}$ and of $M(X, r, y)_{\text {red }}$. Since we apply elementary transformation and elementary antitransformations outside $\operatorname{Sing}(X)$, everything works verbatim if we fix a set $\Omega$ of formal isomorphism types.

Construction 1.3. Fix a formal isomorphism type $\tau=\left\{F_{x}\right\}_{x \in \operatorname{Sing}(X)}$ for rank $r$ torsion-free sheaves around $\operatorname{Sing}(X)$. The existence of a rank $r$ torsion-free sheaf $F$ on $X$ with $\tau$ as formal (or analytic) isomorphism type around $\operatorname{Sing}(X)$ is well known. Here we will show that any such $F$ is a flat limit of stable rank $r$ torsionfree sheaves with $\tau$ as formal isomorphism type around $\operatorname{Sing}(X)$. Fix any rank $r$ torsion-free sheaf $F$ with $\tau$ as formal isomorphism type. If $F$ is stable, we are done. If $F$ is not stable let $u$ be the minimal integer with $0<u<r$ such that there is a rank $u$ saturated subsheaf $A$ of $F$ with $r \chi_{A} \geq u \chi_{F}$. By the minimality of $u$ we see that $A$ is stable. Let $\Sigma$ be the set of all subsheaves of $F$ with $\chi_{A}$ as Hilbert polynomial. Every $B \in \Sigma$ is saturated in $F$. By the minimality of $u$ we see that every $B \in \Sigma$ is stable. As in the proof of Theorem 1.2 we see the existence of $k \geq 1$ and $A_{1}, \ldots, A_{k} \in \Sigma$ such that $G:=A_{1}+\cdots+A_{k} \cong A_{1} \oplus \cdots \oplus A_{k}$ and that every $B \in \Sigma$ is contained in $G$. Now apply elementary transformations and elementary antitransformations around general points of $X_{\text {reg. }}$. If $F \neq G$, we see in this way (as in the proof of Theorem 1.2) $F$ as a flat limit of rank $r$ torsion-free sheaves with $\tau$ as formal isomorphism type, but with no rank $u$ subsheaf with $\chi_{A}$ as Hilbert polynomial. Then we iterate the trick. Since $\chi_{F}$ has only finitely many coefficients, after finitely many iterations we must obtain a stable sheaf, $\Phi$. The proof gives indeed a flat family of such $\Phi$ and $F$ as one of its limits.

Proof of Theorem 0.1. Twisting by a line bundle, we may assume $r\left(2 p_{a}-2\right)+r e<$ $d \leq r\left(2 p_{a}-2\right)+r e+r$, hence $h^{1}(F)=0$ and $F$ is spanned by [Co], Lemma 3.5.6 (or see $[\mathrm{N}]$, Lemma $5.2^{\prime}$, for a somewhat higher bound). Hence we have an exact sequence

$$
0 \rightarrow(r-1) \boldsymbol{O}^{r} \rightarrow F \rightarrow G \rightarrow 0
$$

with $\operatorname{rank}(G)=1$. By [Co], Prop. 2.2.1, for every $x \in \operatorname{Sing}(X)$, the germ $F_{x}$ is isomorphic to a germ, $A_{x}$, with $r \boldsymbol{O}_{X, x} \subseteq A_{x} \subseteq r\left(\pi_{*} \boldsymbol{O}_{C, x}\right)$. Hence for a general choice of the $r-1$ sections we may assume length $(\operatorname{Tor}(G)) \leq r \delta$. Set $G^{\prime \prime}:=$ $\mathcal{H o m}\left(\mathcal{H o m}\left(G, \omega_{X}\right), \omega_{X}\right)$ and $J:=\operatorname{Tor}(G)$. We have $G^{\prime \prime}=G / J$ by the duality 
for torsion-free sheaves. Note that moving $G$ and taking all possible extensions we find all possible $F$ and when $G$ moves in an integral variety with constant $\operatorname{dim}_{\mathbf{K}}\left(\operatorname{Ext}^{1}(G, \boldsymbol{O})\right)$, then we get an integral family of rank $r$ torsion-free sheaves by [BPS]. Since $G^{\prime \prime}=G / J$, we have $\operatorname{dim}_{\mathbf{K}}\left(\operatorname{Ext}^{1}(G, \boldsymbol{O})\right) \leq \operatorname{dim}_{\mathbf{K}}\left(\operatorname{Ext}^{1}\left(G^{\prime \prime}, \boldsymbol{O}\right)\right)+$ $\operatorname{dim}_{\mathbf{K}}\left(\operatorname{Ext}^{1}(J, O)\right)$. Since $J$ is supported on $\operatorname{Sing}(X)$ and has a filtration with as graded pieces sheaves of length 1 and $h^{0}(X, J) \leq r \delta$, we have $\operatorname{dim}_{\mathbf{K}}\left(\operatorname{Ext}^{1}(J, \boldsymbol{O})\right) \leq$ $r \delta \mathbf{f}$. By the vanishing of $H^{1}(F)$ and the local to global spectral sequence of the Extfunctor, $\operatorname{dim}_{\mathbf{K}}\left(\operatorname{Ext}^{1}\left(G^{\prime \prime}, \boldsymbol{O}\right)\right) \leq h^{0}\left(\operatorname{Ext}^{1}\left(G^{\prime \prime}, \boldsymbol{O}\right)\right)+h^{1}\left(G^{\prime \prime *}\right) \leq h^{0}\left(\operatorname{Ext}^{1}\left(G^{\prime \prime}, \boldsymbol{O}\right)\right)+$ $d+2 p_{a}-2 \leq h^{0}\left(\operatorname{Ext}^{1}\left(G^{\prime \prime}, \boldsymbol{O}\right)\right)+(r+1)\left(2 p_{a}-2+e\right)$. By [Co], Lemma 2.2.4, $G_{x}^{\prime \prime}$ is generated by $e_{x} \boldsymbol{O}$. Hence we have an exact sequence

$$
0 \rightarrow U \rightarrow R \rightarrow G^{\prime \prime} \rightarrow 0
$$

with $R$ locally free of rank $e$. Applying $\mathcal{H o m}(-, \boldsymbol{O})$ to the exact sequence (2) and splitting the exact sequence obtained into short exact sequences we obtain:

$$
\begin{aligned}
& 0 \rightarrow G^{\prime \prime *} \rightarrow R^{*} \rightarrow A \rightarrow 0, \\
& 0 \rightarrow A \rightarrow H \rightarrow \operatorname{Ext}^{1}\left(G^{\prime \prime}, \boldsymbol{O}\right) \rightarrow 0
\end{aligned}
$$

with $A$ and $H$ torsion-free of rank $e-1$. By [Co], Lemma 2.2.4, at each singular point $x$ we have $\operatorname{dim}_{\mathbf{K}}\left(H / m_{x} H\right) \leq e_{x}(e-1)$. Hence we have $h^{0}\left(\operatorname{Ext}^{1}(G, \boldsymbol{O})\right) \leq$ $\left(\sum_{x} e_{x}\right)(e-1)=e^{\prime \prime}(e-1)$. Hence the result.

The bound given by Theorem 0.1 is far from being sharp. We do not know any general method to construct irreducible components of $M(r, d)$ or of $M(r, d, \Gamma)$. Hence we have no reasonable guess for the number of components of these moduli spaces.

Remark 1.4. Look at the proof of Theorem 0.1. In that proof we were obliged to fix $\Gamma$ just to control the torsion part $J$ of the sheaf $G$ in eq. (1), its deformations and all its extensions with $G^{\prime \prime}$ to get some sheaf, while the part concerning $G^{\prime \prime}$ alone was (by definition) controlled by $n(1)$. Hence, to get an upper bound for $n(r)$ instead of a bound on the number of irreducible components of the scheme of formal isomorphism types, it is sufficient to control the number of the irreducible components of the scheme of all possible $J$. Note that there is the a priori bound $h^{0}(X, J) \leq r \delta$. For any fixed $x \in \operatorname{Sing}(X)$ and every integer $t \leq r \delta$ the set of all possible $\boldsymbol{O}_{X}$-module structures on the vector space $\mathbf{K}^{t}$ is a scheme $W$ given by degree 2 nonhomogeneous equations (i.e. for every $a \in \boldsymbol{O}_{X}$ the multiplication map $\mu_{a}: \mathbf{K}^{t} \rightarrow \mathbf{K}^{t}, \mu_{a}(m):=a m$ satisfying the condition " $a(b m)=(a b) m$ for all $a, b \in \boldsymbol{O}_{x}$ ") in the affine space $\mathbf{K}^{t}$. By an elementary lemma attributed to A. Andreotti $W_{\text {red }}$ has at most $2^{t}$ irreducible components. Hence in this way we obtain only an exponential bound if we do not fix $\Gamma$. However (and this is the main point), although of exponential type, this upper bound does not depend on the category of torsion-free modules over the rings $\left\{\boldsymbol{O}_{x}\right\}_{x \in \operatorname{Sing}(X)}$. Hence Theorem 0.1 gives an answer to the question of bounding $n(r)$ only in terms of geometric properties of $\operatorname{Sing}(X)$ and the integer $n(1)$ (i.e. the rank 1 case).

\section{REFERENCES}

[BPS] C. Banica, M. Putinar, G. Schumacher, Variation der globalen Ext in Deformationen kompakter komplexer Räume, Math. Ann. 250 (1980), 135-155. MR 82e:32015

[Co] P. Cook, Local and global aspects of the module theory of singular curves, Ph.D. Thesis, Liverpool 1993. 
[M] M. Maruyama, Elementary transformations of algebraic vector bundles, in: Algebraic Geometry - Proceedings La Rabida, pp. 241-266, Lecture Notes in Math. 961, Springer-Verlag, 1981. MR 85b:14020

[N] P. E. Newstead, Introduction to Moduli problems and Orbit Spaces, T.I.F.R. Lecture Notes 51, 1978. MR 81k:14002

[S] C. S. Seshadri, Fibrés vectoriels sur les courbes algébriques, Astérisque 96, 1982. MR 85b: 14023

Department of Mathematics, University of Trento, 38050 Povo (TN), Italy

E-mail address: ballico@science.unitn.it 